\title{
Interconnection Model and Mobility Scenarios in the Context of Handover Satellite
}

\author{
S. Aris ${ }^{1}$, S. Sadouni ${ }^{1}$, J. L. Beylot ${ }^{2}$, M. Benslama ${ }^{1}$ \\ ${ }^{1}$ Electromagnetic and Telecommunication Laboratory, University of MENTOURI Brothers, \\ Faculty of Science Technology. Constantine 1 Algeria \\ ${ }^{2}$ INPT/ENSEEIHT, IRIT - enseeiht.fr, Toulouse France
}

\begin{abstract}
In the field of telecommunications, mobility is the new mode of communication used to offer a great flexibility of use for its various users who require a continuous connection with a good quality of service. In this paper, we propose to integrate the concept of Handover with satellite networks, in order to increase their robustness, their resistance to mobility effects and the optimization of strategies in satellite network resources. To do this, we have chosen the SCTP/IP protocol in a DVB-S/RCS satellite type system, which will support the introduction of the multihoming concept on MANET (Ad Hoc mobile networks) networks, and make it co-exist with other Ad Hoc routing protocols.
\end{abstract}

Keywords: DVB-S/RCS, Handover, SCTP/IP, MANET, Multihoming.

\section{Introduction}

In recent work, we have discussed the processing of SCTP protocols in mobile networks $[1,2,3]$. On the basis of the results obtained, we extend our study to the case of handover in satellites. WiMAX Mobile and Satellite interconnection with norm DVB-S/RCS is formed of a WiMAX cell with a coverage radius of $3 \mathrm{kmas}$ well as a zone covered by a geostationary satellite with a radius of $50 \mathrm{Km}$.

The intersection of these two cells forms a Handover (HO) zone, by passing through this zone the MS should be able to connect with both networks at the same time. The latter's main purpose will be ULE /MPEG2TS encapsulation or de-encapsulation of the digital IP packets that it receives or transmits, as well as access routing for the upstream traffic (attribution of priority levels to IP flows on return and appropriate processing). The satellite responsible for diffusing the traffic at the MS in the coverage zone communicates directly with a NCC Gateway on the ground and in turn is connected to a router than ensures internet access.

As for the WiMAX network, the BS is connected to a ASN-GW and the latter is linked to the CSN, which provides direct access to an Internet network. copy the image to the Windows clipboard and then Edit | Paste Special | Picture (with "Float over text" unchecked).

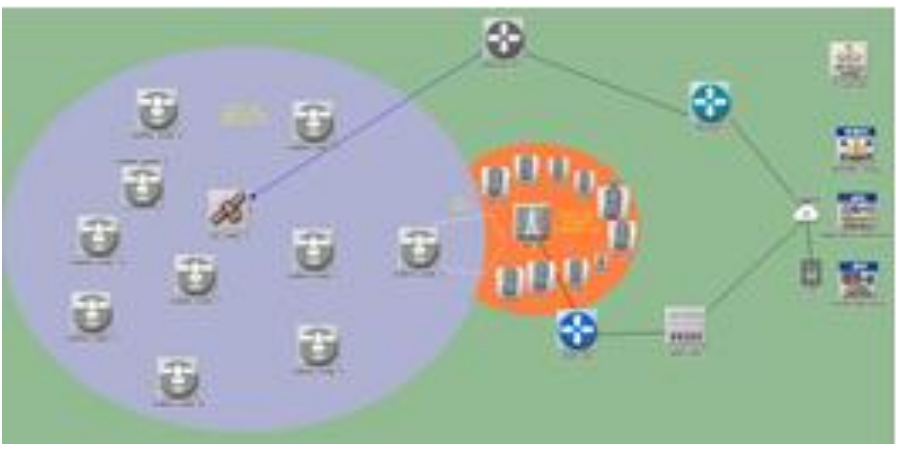

Fig. 1 Model for interconnecting WiMAX Mobile Satellite 


\section{Simulation Scenarios}

The aim of this study is to define the version of the SCTP protocol that ensures a better Qos during passage of the MS from one satellite network to another WiMAX Mobile with complete transparency and vice versa. To do this, the MS should make several journeys from the satellite network to WiMAX and back throughout the entire simulation. Since the network resources are limited, to get closer to reality we install several other mobile stations in the satellite and WiMAX coverage zones. These mobile stations start to communicate with their respective networks 20 minutes from the start of the simulation. We also program a traffic generating application between the MS and CN to transit a maximum flow of $12000 \mathrm{bit} / \mathrm{sec}[4,5,6]$.

With the aim of validating our work's qualitative contribution, we carry out a comparative study between three different scenarios.

- The first scenario: The MS and its correspondent (CN) use SCTP as a transport protocol.

- Second scenario: The MS and CN use MSCTP as a transport protocol.

- Third scenario: The MS and CN use CSCTP as a transport protocol.

To show the impact of increasing the speed on the MS's performances, it moves at two speeds: 50 and 100 $\mathrm{km} / \mathrm{h}$.

\subsection{Implementation with Simulation}

In the first and second chapters, we respectively activate the SCTP and MSCTP protocols at transport layer 4.

We return to the same modifications carried out in the previous chapters for installing SCTP and MSCTP in the Simulator, for MS and CN [7,8].

For the third scenario in layer 4, we program the CSCTP protocol. To do this, we add a new Handoff state to the MSCTP's operation, see figure 8.15 whose main function is to inform the $\mathrm{CN}$ when a handoff mode commences at the MN. This modification is made with the aim of quickly indicating the change of address to the $\mathrm{CN}$. The CN considers both the known addresses (the old primary address and the new, added address) as the primary address; in this case, the $\mathrm{CN}$ sends duplicated packets on the two primary addresses to the MN. The association's old IP address is deleted when the MN decides that an address is inactive.

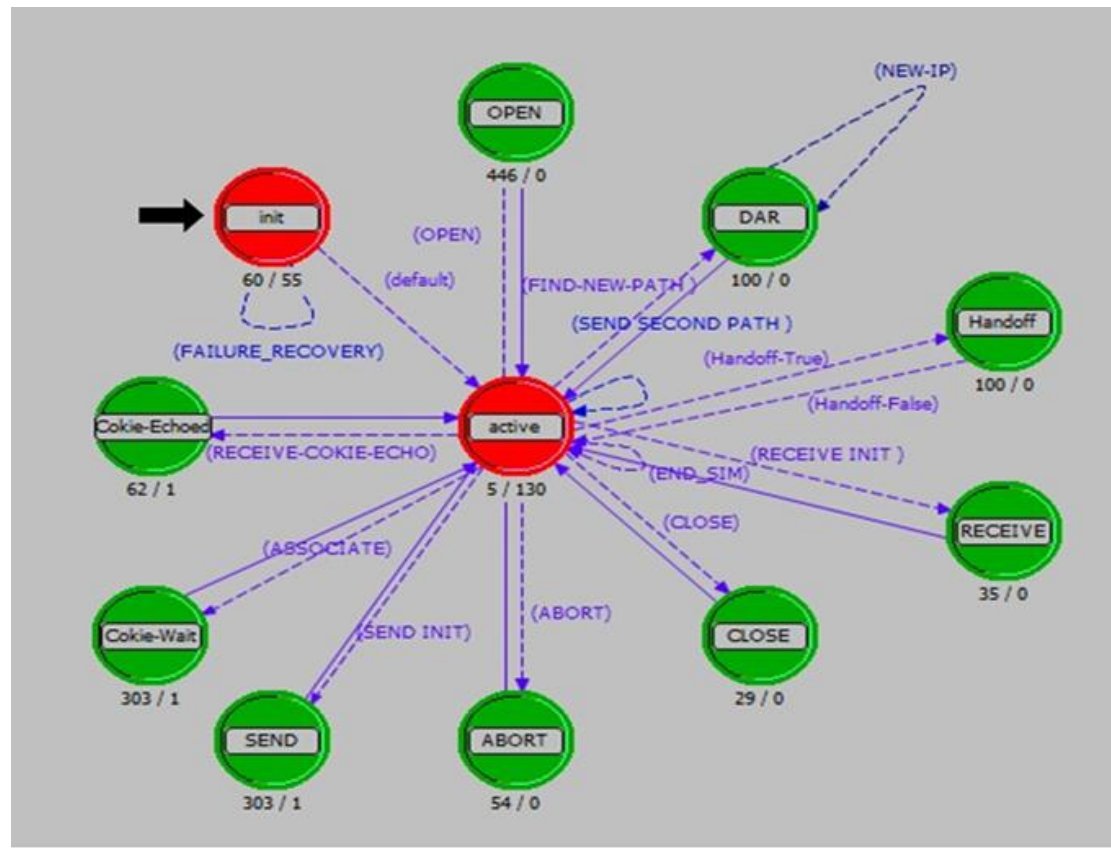

Fig. 2 Process Model of the CSCTP Protocol 
Finally, to ensure correct running of the simulation, the MS's and CN's network interfaces should both install the same transport protocol for each scenario. Since the MS moves between the two WiMAX Mobile and satellite networks, this means that activation of the transport protocol in the scenario occurs on the two communication interfaces. We take the example of satellite network interfaces used in the third scenario, based on the CSCTP protocol, see figure 3.

To enable the MS to receive and transmit packets supporting the SCTP/IP protocol in a DVB-S/RCS satellite type system, the data packets should be type MPEG2-TS as we have already mentioned above. Solving this difficulty means using an encapsulation mechanism at the link layer.

According to $[9,10]$ the ULE encapsulation mechanism is most appropriate for satellite communications, with SCTP/IP type flows.

Encapsulating SCTP packets by ULE creates another MPEG2-TS type packet to be sent by the physical layer DVB-S from the NCC gateway to the satellite, and by the DVB-RCS physical layer from the MS's satellite interface (see figure 3).

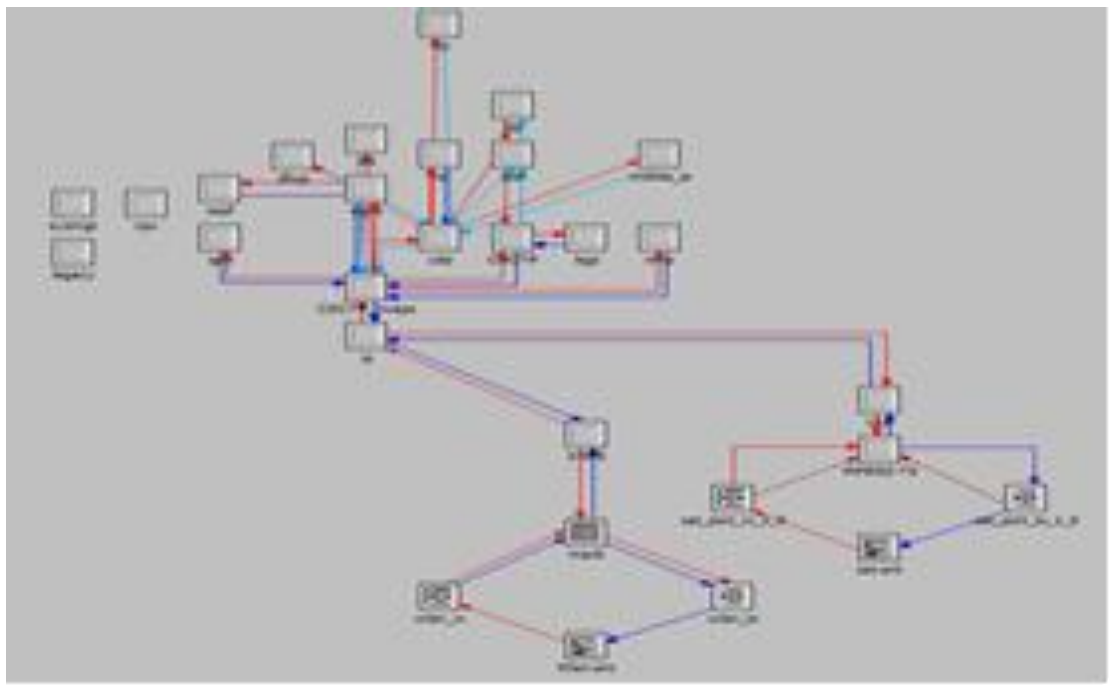

Fig. 3 Satellite interface using the CSCTP protocol, scenario 3

\subsection{Simulation parameters for the satellite network}

TABLE I: Simulation Parameters for the Satellite Network

\begin{tabular}{|c|c|}
\hline \multicolumn{2}{|c|}{ Sat-node parameters } \\
\hline Orbit & Low-earth \\
\hline altitude $(\mathrm{km})$ & 1000 \\
\hline Cycle time & 60 \\
\hline Jammer band base frequency & 30 \\
\hline Jammer bandwidth & 10 \\
\hline Jammer transmitter power & 100 \\
\hline Number of frequencies \\
\hline \multicolumn{2}{|c|}{ Gateway NCC } \\
\hline Processing Scheme & Central Processing \\
\hline $\begin{array}{c}\text { Datagram switching rate } \\
\text { (pkt/sec) }\end{array}$ & 500000 \\
\hline Datagram forwarding rate & 150000 \\
\hline Maximum Static Routes & 4000 \\
\hline Timeouts SCTP MSCTP \\
CSCTP
\end{tabular}




\begin{tabular}{|c|c|}
\hline \multicolumn{2}{|c|}{ Mobile Node } \\
\hline $\begin{array}{c}\text { Wireless lan MPE2-TS data } \\
\text { rate (Mbps) }\end{array}$ & 11 \\
\hline Transmit Power (w) & 0.005 \\
\hline $\begin{array}{c}\text { Packet Reception Power } \\
\text { threshold (dBm) }\end{array}$ & -95 \\
\hline Buffer size (bits) & 256000 \\
\hline Large packet processing & Drop \\
\hline Long retry limit & 7 \\
\hline Short retry limit & 4 \\
\hline
\end{tabular}

For the WiMAX Mobile network, we use the simulation parameters already mentioned (see table 1)

\subsection{Performance criteria}

To compare the performances offered by the SCTP, MSCTP and CSCTP protocols in vertical handover between the WiMAX Mobile and Satellite, we suggest the mean output received by the MS as performance criteria.

\subsection{Results}

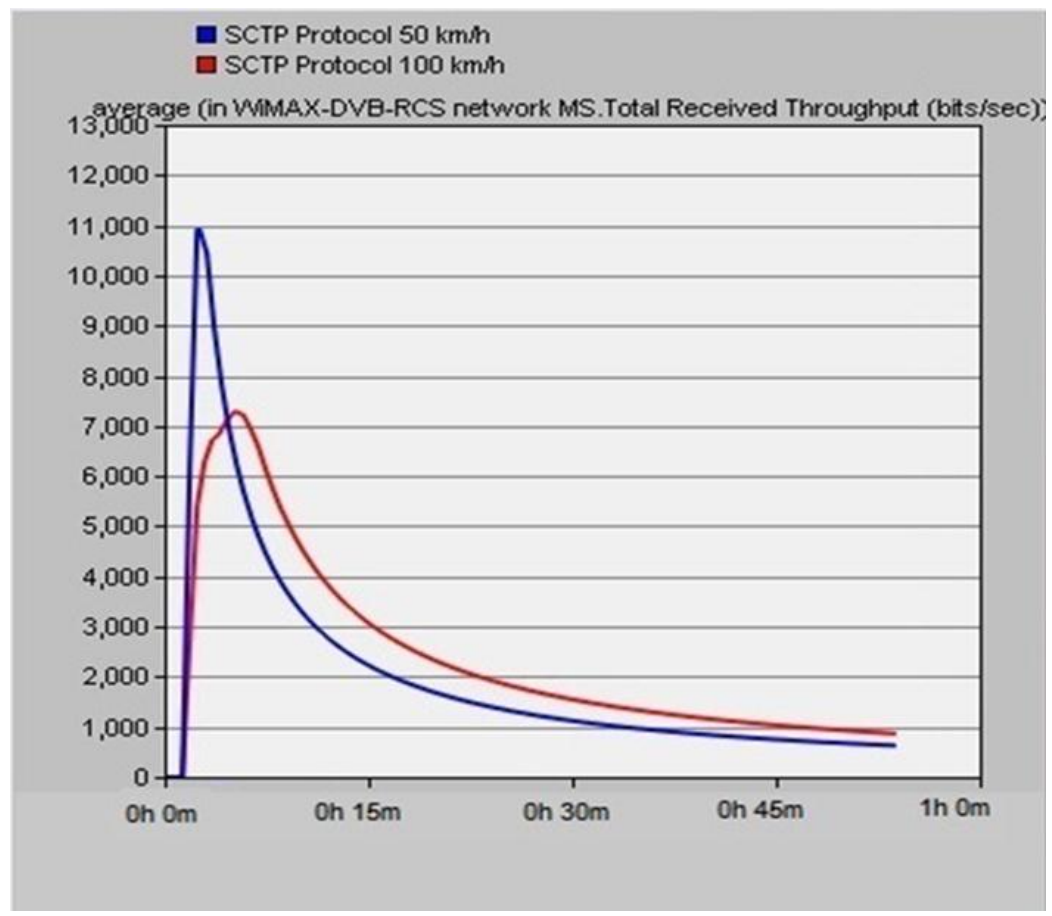

Fig. 4 The mean output for the SCTP protocol for speeds of $50 \mathrm{~km} / \mathrm{h}$ and $100 \mathrm{~km} / \mathrm{h}$

On figure 4 we see the mean output received by the MS in bit/sec, using the SCTP transport protocol (scenario 1). So we see that using this protocol creates a significant rise in the output, which reaches 11000 $\mathrm{bit} / \mathrm{sec}$ for a speed of $50 \mathrm{~km} / \mathrm{h}$ and $7000 \mathrm{bit} / \mathrm{sec}$ for $100 \mathrm{~km} / \mathrm{h}$ at the start of the simulation. Then the output drops very rapidly to below $900 \mathrm{bit} / \mathrm{sec}$ at the end of the simulation as we mentioned before, the network traffic generator application transmits a flow of $12000 \mathrm{bit} / \mathrm{sec}$ between the $\mathrm{CN}$ and the MS. Nevertheless, the version based on the SCTP protocol reach the maximum flow only at the start of the simulation, for a very short time.

Consequently, we will henceforth concentrate on using the MSCTP protocol as well as the CSCTP protocol. 


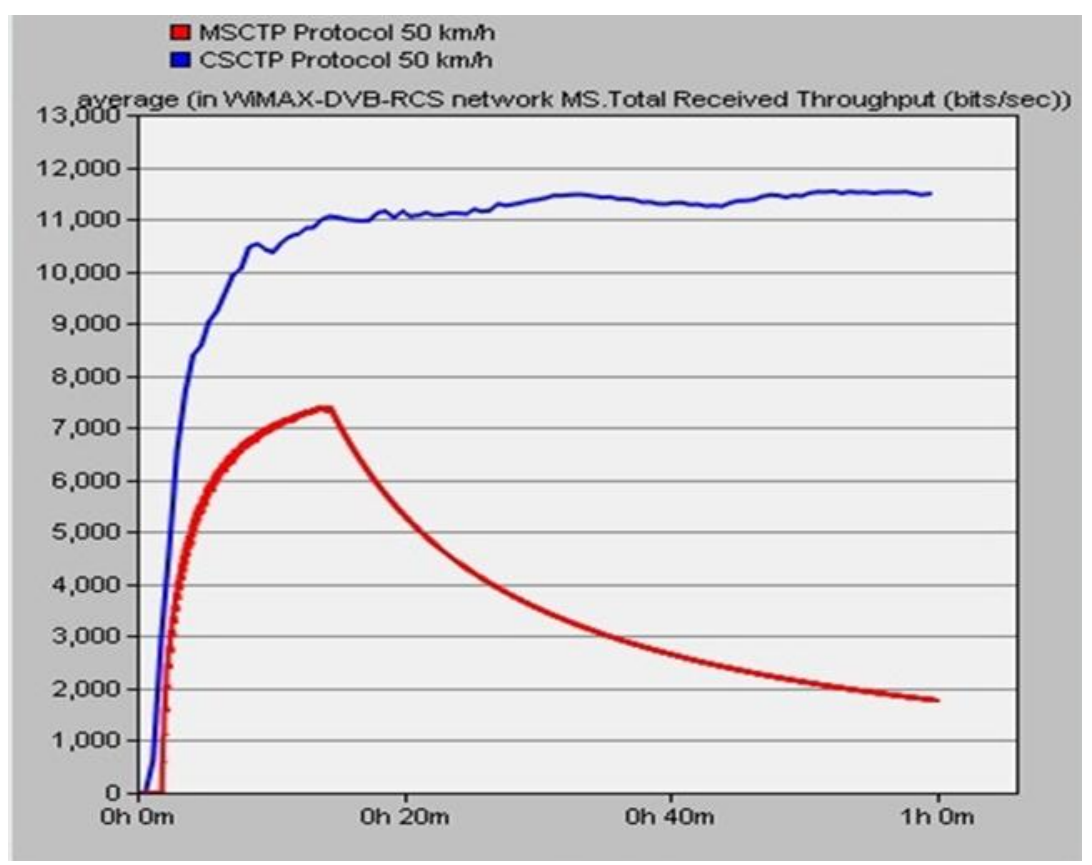

Fig. 5 The mean output for the MSCTP and CSCTP protocols for a speed of $50 \mathrm{~km} / \mathrm{h}$

Figure 5 shows a comparison between the mean output received by the MS compared to scenario 2 (using the MCTP) and scenario 3 (using CSCTP). This comparison is made using a speed of $50 \mathrm{~km} / \mathrm{h}$, and we see that the two scenarios show differences in output from the start of the simulation. This difference in output increases very quickly with time. However, after the 18th minute of simulation, the output using MSCTP protocol starts to decrease to eventually drop below $2000 \mathrm{bit} / \mathrm{sec}$.

The output from the CSCTP however continues to increase to reach a maximum value of $11563 \mathrm{bit} / \mathrm{sec}$ at the end of the simulation.

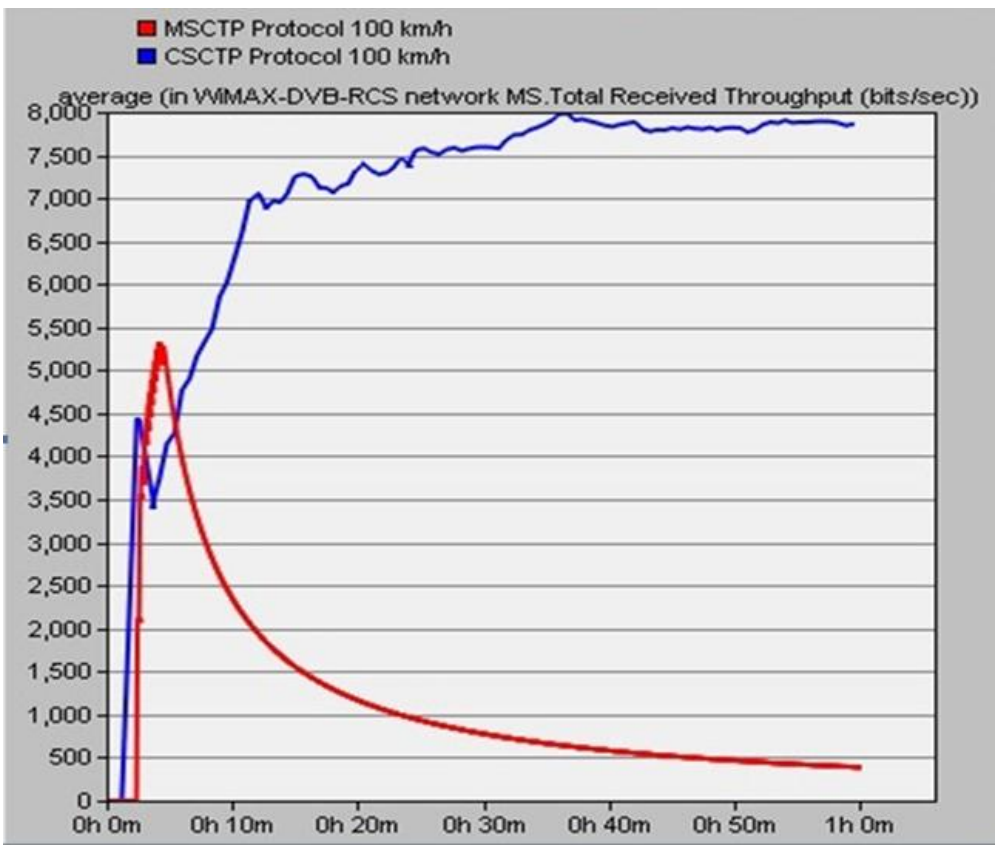

Fig. 6 Mean output from the MSCTP and CSCTP protocols for a speed of $100 \mathrm{~km} / \mathrm{h}$

Similarly, figure 6 shows a comparison between the mean output received by the MS compared to scenario 2 (using MSCTP) and scenario 3 (using CSCTP). However, this comparison is made using a speeed of $100 \mathrm{~km} / \mathrm{h}$, 
and we see that the two scenarios present differences in output from the start of the simulation. MSCTP reaches a peak output with a value of $5398 \mathrm{bit} / \mathrm{sec}$, to then drop very quickly to below $500 \mathrm{bit} / \mathrm{sec}$. However, CSCTP continues to increase to reach a maximum value of $8000 \mathrm{bit} / \mathrm{sec}$.

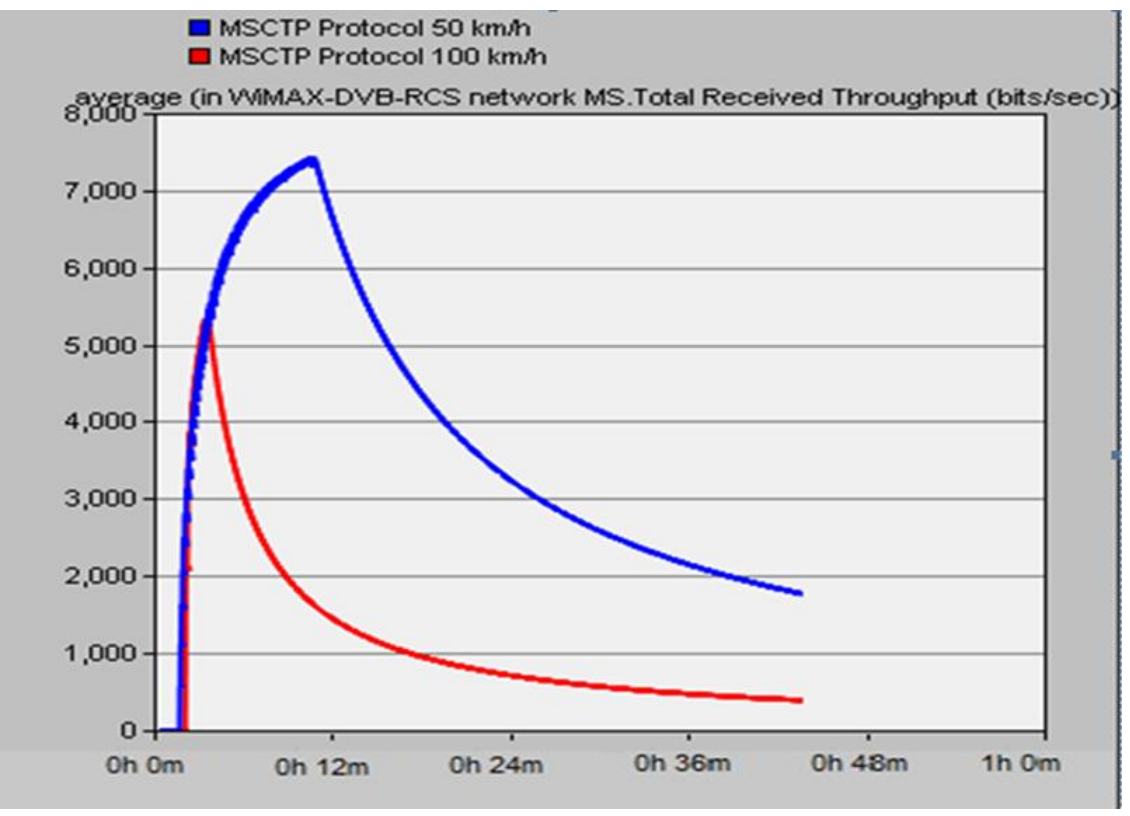

Fig. 7 Comparison of mean output from the MSCTP protocol for a speed of 50km/h and $100 \mathrm{~km} / \mathrm{h}$

Figure 7 shows a comparison between the mean output received by the MS compared to scenario 2, for speeds of 50 and $100 \mathrm{~km} / \mathrm{h}$. Even if the effects of the speed are visible from the start of the simulation, the two variants of this scenarios show a relatively low output. This output reaches a maximum at the start of the simulation, to then drop below $2000 \mathrm{bit} / \mathrm{sec}$ for a speed of $50 \mathrm{~km}$ and $500 \mathrm{bit} / \mathrm{sec}$ for a speed of $100 \mathrm{~km} / \mathrm{sec}$ at the end of the simulation.

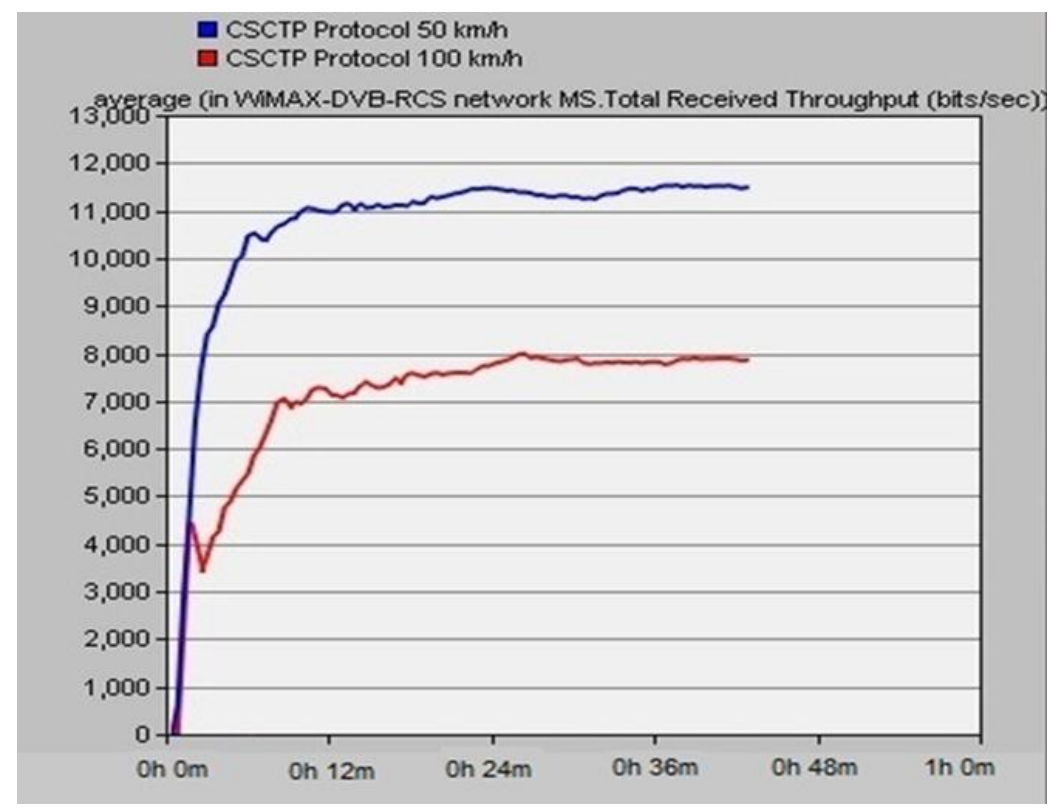

Fig. 8 Comparison of the mean output from the CSCTP protocol for speeds of $50 \mathrm{~km} / \mathrm{h}$ and $100 \mathrm{~km} / \mathrm{h}$

As the CSCTP protocol presents a better output than SCTP and MSCTP, on figure 8 we compare the output of an MS installed on CSCTP and moving with a speed of $50 \mathrm{~km} / \mathrm{h}$ and $100 \mathrm{~km} / \mathrm{h}$. We see the effects of the 
speed from the start of the simulation, as the two variants of scenario 3 have the same value only for a few seconds. Then, the difference in output between the two speeds widens; for a speed of $50 \mathrm{~km} / \mathrm{h}$ the output registers a maximum value of $8000 \mathrm{bit} / \mathrm{sec}$ whereas with a speed of $100 \mathrm{~km} / \mathrm{h}$ it reaches $11563 \mathrm{bit} / \mathrm{sec}$.

\section{Discussion of the Results}

In a WiMAX mobile and satellite during a vertical handover networks, the transport protocol plays a fundamental role for creating transparency of passage during a vertical handover between the two networks. We have seen this by comparing the different scenarios shown in this chapter. As we have already noted, SCTP and MSCTP are not reliable protocols for a vertical handover between WiMAX Mobile and DVB-RCS Satellite, as the present reliable flows that are aggravated by the effect of increasing the speed, as well as the number of subscribers in the two networks' coverage zones.

As for CSCTP, this protocol has proven to be very resistant to the effects of a vertical Handover between WiMAX and Satellite networks as it achieves a satisfactory mean output. Nevertheless, increasing the speed of movement involves decreasing the mean output by nearly $33 \%$.

We explain these results by the speed with which the $\mathrm{CN}$ is informed when a handoff mode is triggered by the MN using the chunks flags' Hbit. This modification enables the CN to consider simultaneously both the paths defined by the MN (the old and the new) as primaries.

In this case, the $\mathrm{CN}$ sends duplicated packets on the two primary addresses to the MN. With the substantial delays satellite communications involve, for MSCTP the data packets are sent from the old IP address before the $\mathrm{MN}$ considers the new IP address as a primary address for the association underway.

\section{Conclusion}

Due to the great difference in performance between the WiMAX Mobile and Satellite systems, interconnection between them has proven to be very complex. In this chapter we have suggested an interconnection model between a spatial network based on the principle of diffusion by satellite, and formed of two combined systems: the DVB-S/RCS and a WiMAX Mobile network. With the aim of achieving transparent passage between these two systems with the both constraints of speed and of the number of subscribers, we have based our study on the use of three transport protocols: SCTP MSCTP and CSCTP

The results of simulations obtained from our model show that the CSCTP transport protocol is better adapted for a vertical Handover between WiMAX Mobile and Satellite networks. Thus, use of the Multihoming concept is more favorable for the output in the context of using the CSCTP protocol than for the MSCTP protocol, which suggests results comparable to those of the SCTP.

\section{References}

[1] S. Sadouni, M. Benslama, A. Mebarki, A. Beylot: SCTP-WSN new extension for more reliable sparse wireless sensor networks . 2017 13th International Wireless Communications and Mobile Computing Conference (IWCMC) , p. 2109 2114, 2017

[2] S. Sadouni, M. Benslama, A. L. Beylot: New SCTP protocol extension for more robust Ad Hoc mobile networks. 2016 4th International Conference on Control Engineering \& Information Technology (CEIT),p. 1 - 6, 2016,

[3] S. Sadouni, M. Benslama, A. L. Beylot: Increasing the performances of UMTS and WIMAX heterogeneous mobile networks by the integration of Multihoming. 2016, 4th International Conference on Control Engineering \& Information Technology (CEIT), p. 1 - 6, 2016,

[4] L.Xu, P.Wang, Q.Li, et al': Call admission control with inter network cooperation for cognitive heterogeneous networks. IEEE Transactions on Wireless Communications, Vol. 16, n 3, pp.1963 - 1973, 2017.

[5] H. Zhang, P.Dong, S.Yu, et al': A scalable and smart hierarchical wireless communication architecture based on network/user separation. IEEE Wireless Communications, Vol. 24, n 1, pp. 18 - 24, 2017. 
[6] R. Ravindran, A. Chakraborti, S.O.Amin, et al': 5G -ICN delivering ICN services over 5 G using network slicing. IEEE Communications Magazine, Vol. 55, n 5, pp.18-24, 2017.

[7] J. R. Iyengar, P.D. Amer, R.Stewart: Concurent multipath transfer using SCTP multihoming over independent end to end paths. IEEE/ACM Transactions on Networking, Vol.14, n 5, pp.951-964, 2006.

[8] M.Watari, T.Ernest, R.Wakikawa, et al': Routing optimization for nested mobile networks, special section on mobile multimedia communications. IEICE Trans. Comm., Vol. E89-B, n¹0, 2006.

[9] S. Corson and J. Macker: Mobile Adhoc networking (MANET): Routing protocol Performance Issues and Evaluation Considerations, RFC 2501, 1999.

[10] C. Cathern et al': CISCO Networks A Press 2015. 